\title{
LA MUCORMYCOSE NASOSINUSIENNE DIAGNOSTIC ET MODALITES THERAPEUTIQUES
}

\author{
I. LAHMAR, S. JERBI ${ }^{*}$, H. CHAHED, R. FDHILA, K. MIGHRI, L. NJIM**, \\ F. BEN ROMDHANE ${ }^{\star * \star}$, N. DRISS. \\ SERVICE D'ORL, HÔPITAL DE MAHDIA. TUNISIE \\ * SERVICE D'IMAGERIE MÉDICALE, HÔPITAL DE MAHDIA. TUNISIE \\ ** SERVICE D'ANATOMIE CYTOLOGIE PATHOLOGIQUE, HÔPITAL DE MONASTIR. TUNISIE \\ *** SERVICE DE MALADIES INFECTIEUSES, HÔPITAL DE MONASTIR. TUNISIE
}

\begin{abstract}
La mucormycose est une infection fongique rare qui touche essentiellement les sujets immunodéprimés et notamment diabétiques. La localisation de cette maladie est surtout nasosinusienne. Son pronostic reste mauvais malgré le développement des moyens de prise en charge.

Nous rapportons deux cas de mucormycose nasosinusienne à travers lesquels nous discutons les aspects cliniques et radiologiques, ainsi que les moyens thérapeutiques de cette maladie.

II s'agit d'un homme et d'une femme âgés respectivement de 56 et 52 ans. Le premier était diabétique et la deuxième insuffisante rénale. L'évolution était lente dans le premier cas et très rapide dans le deuxième. Le diagnostic était dans les deux cas histologique. L'évolution était, dans le premier cas, favorable après traitement associant débridement chirurgical et amphotéricine $\mathrm{B}$, et dans le second rapidement fatale.

Conclusion : La mucormycose nasosinusienne est une affection grave dont le pronostic peut être mauvais malgré le traitement.

Mots clés : Infection fongique, mucormycose rhinocérébrale, zygomycètes.
\end{abstract}

\section{SUMMARY}

Mucormycosis is a rare fungal infection which affects immunocompromized especially diabetic subjects. Rhino maxillary is its most common location. Although development of treatment this illness have a poor prognosis.

We report two cases of rhino maxillary mucormycosis and study clinical manifestations, radiologic findings and treatment of this dangerous affection. They were a 56 years old man and a 52 years old woman. The man had diabetes and the women a chronic renal insufficiency. Disease evoluated since one month in the first case and six days in the second.

Diagnostic was obtained by histopathologic study in two cases. After surgical and medical treatment the first patient has survived. The second has died before beginning treatment

Conclusion : Mucormycosis is a rare affection which can have lethal evolution, even after adequate treatment Keywords : Fungal infection, rhinocerebral mucormycosis, zygomycetes.

\section{INTRODUCTION}

La mucormycose est une infection fongique rare. C'est une maladie grave associée à un taux de mortalité élevé. Elle touche les terrains immunodéprimés et essentiellement les diabétiques en décompensation acidocétosique. Transmise par voie aérienne, elle a un tropisme essentiellement nasosinusien. Néanmoins, d'autres localisations : digestive, pulmonaire et cutanée sont possibles, mais beaucoup plus rares.

\section{OBSERVATIONS}

\section{Observation 1 :}

Le patient Mustapha $C$. âgé de 56 ans, diabétique depuis 10 ans, a été hospitalisé en Octobre 1996 pour un œdème hémifacial gauche évoluant depuis 1 mois avec, 10 jours avant I'hospitalisation, une obstruction nasale, des paresthésies hémifaciales et une exophtalmie homolatérales. L'examen a objectivé un œdème de l'hémiface gauche ainsi qu'une paralysie oculomotrice et une exophtalmie du même côté. La fosse nasale gauche était imperméable, à muqueuse très inflammatoire et siège de secrétions purulentes au niveau du méat moyen.
Un scanner du massif facial a donc été réalisé. II a montré un comblement du sinus maxillaire gauche, étendu à l'ethmoïde et à l'orbite du même côté ainsi qu'au sinus sphénoïdal (figures 1, 2).

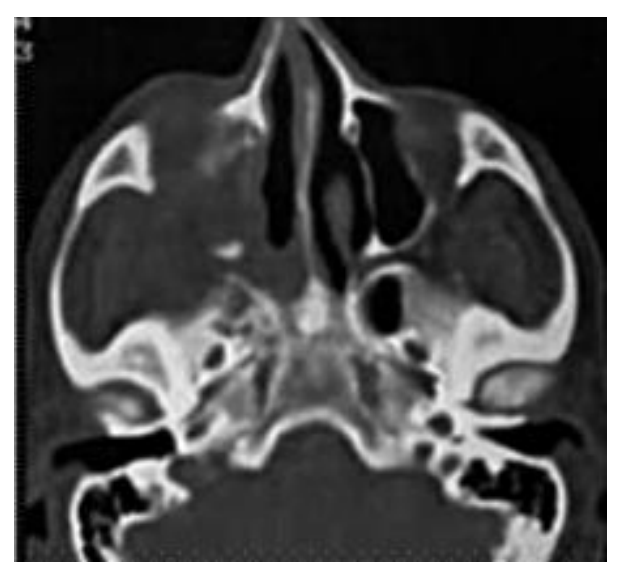

Fig. 1 : Tomodensitomètrie en coupe axiale : comblement total du sinus maxillaire gauche siège d'une opacité calcique avec lyse osseuse 


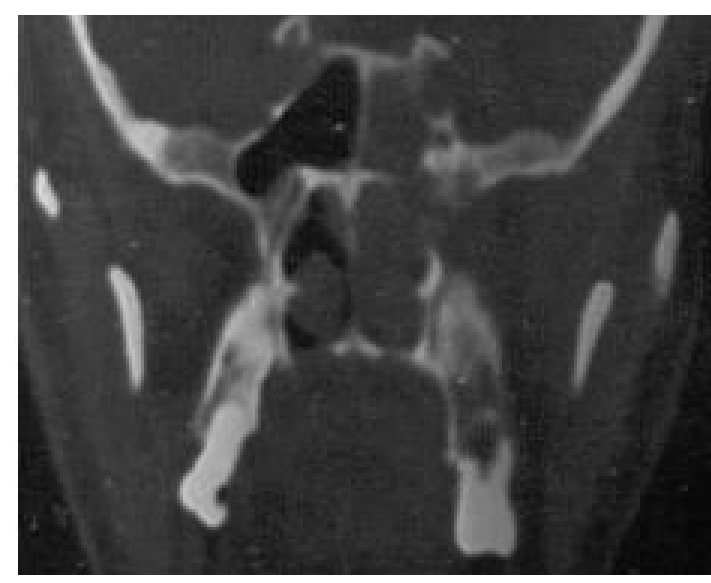

Fig. 2 : Tomodensitomètrie en coupe coronale : comblement total du sinus sphénoïdal gauche

Une biopsie de la muqueuse nasale a été réalisée (au niveau du méat moyen gauche). Elle a conclu à une mucormycose.

Un débridement chirurgical par voie para-latéro-nasale a donc été réalisé, suivi d'un traitement médical par " amphotéricine $\mathrm{B}$ » à raison d'un milligramme par kilogramme par jour durant 5 semaines.

L'évolution était favorable ; le suivi clinique n'a pas montré d'anomalie et une tomodensitomètrie de contrôle fait en 2003 était normal (figure 3). Le dernier contrôle clinique remonte à 2006 (soit un recul de huit ans).

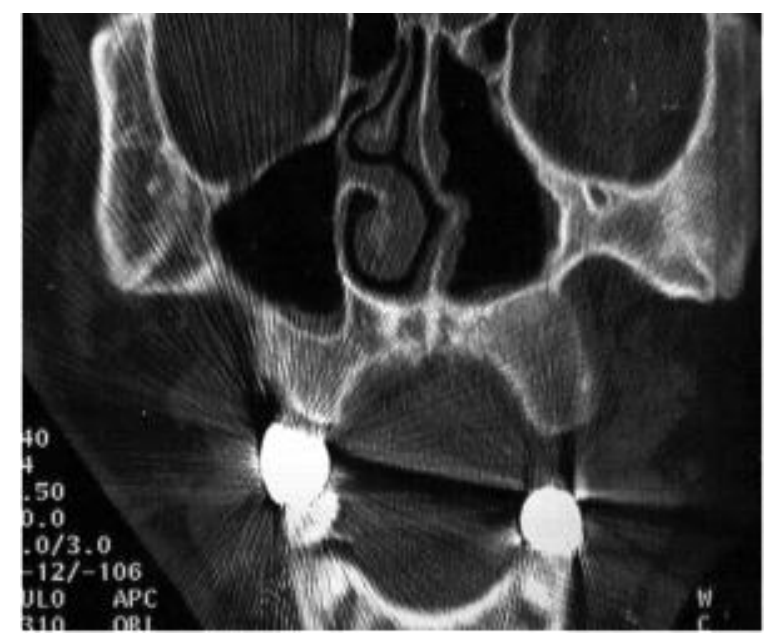

Fig. 3 : Tomodensitomètrie du massif facial en coupe coronale 5 ans après traitement médico chirurgical : Aspect normal

\section{Observation 2 :}

La patiente Aouicha $B$. âgée de 52 ans, insuffisante rénale chronique, a été hospitalisée en Avril 1998 pour tuméfaction jugale gauche évoluant depuis 6 jours dans un contexte fébrile avec nécrose progressive.
A l'examen, elle avait une fièvre à $39,5^{\circ} \mathrm{C}$ ainsi qu'un placard nécrotique jugal gauche étendu à la paupière inférieure du même côté et à l'arête nasale, avec œdème de I'hémiface gauche (figure 4). La patiente avait aussi un aspect nécrotique de la muqueuse nasale. La biopsie a conclu à une mucormycose. L'évolution était très rapidement fatale avant d'entamer le traitement.

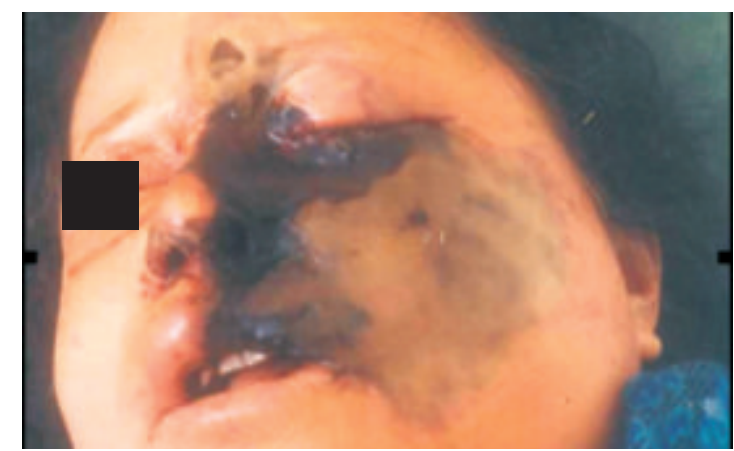

Fig. 4 : Placard nécrotique de l'hémiface gauche

\section{DISCUSSION}

Le premier cas de mucormycose a été décrit en 1889 par Paultauf (in 1). II s'agit d'une infection fongique opportuniste rare (2), dont l'incidence aux Etats Unis est estimée à 500 nouveaux cas par an (3).

Elle est due à des germes d'ordre mucorale qui sont des organismes opportunistes, saprophytes, présents dans les aliments moisis et dans le sol (1, 4). La contamination, quoiqu'elle puisse se faire par voie digestive et cutanée, est essentiellement aérienne faisant de la localisation nasosinusienne la forme la plus commune (4). Le genre causal est dans $70 \%$ des cas le rhizopus. Plus rarement, la maladie est due à d'autres genres comme : absidia, rhizomucor, mucor et actinomucor elegans $(5,6)$.

Les sinus maxillaires et éthmoïdaux sont les localisations électives pour le développement et la prolifération mucorale (5). De là, Les hyphes mycéliens vont envahir les structures vasculo-nerveuses (surtout artériolaires), occasionnant des thromboses vasculaires multiples et des nécroses tissulaires étendues en aval $(4,5,6)$.

La mucormycose, sauf quelques rares cas (7), affecte essentiellement les terrains immuno-déficients suite à une hémopathie, une chimiothérapie, une corticothérapie au long cours, un traitement par la deféroxamine, une neutropénie sévère ou prolongée, une transplantation d'organe, ou une greffe de moelle $(5,6)$.

La mucormycose nasosinusienne est plutôt favorisée par l'acidocétose diabétique. Le mécanisme de cette association est encore mal élucidé : les études ont montré que l'acidose et I'hyperglycémie entrainent une élévation du taux de fer sérique libre (par la diminution de l'affinité de la transferrine au fer) ainsi qu'une diminution du chimiotactisme et du pouvoir phagocytaire des cellules mononuclées et polynucléés, favorisant ainsi la prolifé- 
ration mycosique. Ces constatations pourraient expliquer la fréquence de l'association mucormycose et acidocétose mais pas l'affinité nasosinsienne de la mucormycose en cas d'acidocétose diabétique (3).

L'infection peut commencer par une rhinite ou une sinusite d'apparence banale, évoluant cependant rapidement vers l'extension locorégionale et l'apparition d'une nécrose tissulaire avec éventuellement émission de pus noirâtre évoquant du sang cailloté (8). Le tableau clinique associe une fièvre ( $44 \%$ des cas) ; une nécrose tissulaire endonasale ( $38 \%$ des cas), un œdème orbitaire ou facial (34\% des cas), des troubles visuels (30\% des cas) des céphalées ( $25 \%$ des cas) et des algies facio-orbitaires (22\% des cas) (9).

L'imagerie peut faire évoquer le diagnostic de mucormycose nasosinusienne sans pour autant le confirmer. En effet le scanner du massif facial montre le plus souvent un épaississement de la muqueuse sinusienne sans anomalie de la paroi osseuse. Néanmoins, la découverte d'une lyse des parois osseuses sinusiennes, dans un contexte clinique évocateur, est fortement évocatrice d'une mucormycose nasosinusienne(10). L'imagerie par résonance magnétique (IRM) peut être utile en cas de suspicion d'extension endocrânienne, orbitaire ou au niveau du sinus caverneux $(1,10)$.

II n'y a, actuellement, aucune sérologie fiable, l'hémoculture est souvent négative (3). La confirmation diagnostique peut être faite sur l'examen mycologique du prélèvement nasal à un stade précoce. Si cet examen est négatif une biopsie précoce et profonde des tissus infectés permet d'objectiver des hyphes mycéliens épais courts non septés avec des ramifications à angle droit. La mise en culture permet de déterminer le genre et l'espèce mucorale mais une culture négative est une situation qui n'est pas rare et ne permet en aucun cas d'infirmer le diagnostic de mucormycose nasosinusienne (4). La mucormycose nasosinusienne est une maladie grave. Le retard diagnostic et thérapeutique expose inéluctablement à des complications sévères voire même fatales. En effet, l'infection mycosique peut s'étendre en avant vers la peau qui, initialement d'aspect normal, devient érythémateuse et œdémateuse puis violacée pour finir par se nécroser en laissant des séquelles esthétiques indélébiles (4). L'extension en bas vers la cavité buccale est révélée par des ulcérations nécrotiques au niveau du palais dur. L'extension postérieure peut être source soit de thrombose du sinus caverneux, soit de mucormycose rhino cérébrale (4).

L'atteinte ophtalmologique peut se traduire par une simple conjonctivite, un ptosis, un chémosis, voire même une ophtalmoplégie, un syndrome de l'apex orbitaire, une cécité, ou une occlusion de l'artère centrale de la rétine. La bilatéralisation de ces signes doit faire craindre une thrombose du sinus caverneux qui pourra mettre en jeu le pronostic vital du patient (11).
La mucormycose rhino-cérébrale, complication la plus grave, doit être impérativement recherchée devant un écoulement nasal unilatéral sanguinolent, une atteinte des paires crâniennes (surtout I, V, VII) ou de signes neurologiques déficitaires (5). Elle est en rapport avec des infarcissements cérébraux parfois mortels, qui justifient la mise en route précoce du traitement avant même le résultat de la culture.

Des accidents hémorragiques foudroyants et mortels peuvent survenir en cas d'extension vers les structures vasculaires, essentiellement l'artère carotide interne (8). Le traitement adéquat d'une mucormycose nasosinusienne doit comprendre, en plus du contrôle des facteurs favorisants et l'équilibration des tares, deux volets : un volet chirurgical et un volet médical.

En effet, outre la résistance élevée de certaines souches mucorales à l'amphotéricine B (3), les thromboses vasculaires et la nécrose tissulaire constituent des contraintes qui limitent l'efficacité du traitement médical et qui justifient la nécessité d'un traitement chirurgical adjuvant. L'exploration chirurgicale consiste en un débridement précoce, répété, et complet de la totalité du tissu nécrosé afin d'optimaliser l'effet du traitement médical. En matière de mucormycose rhinocérébrale, la mortalité en cas de traitement antifongique exclusif est estimée à $70 \%$ versus $14 \%$ en cas de traitement chirurgical adjuvant (3).

L'amphotéricine $B$ constitue le traitement médical princeps de la mucormycose nasosinusienne. La forme liposomale, moins nephrotoxique que la forme desoxycholique, a permis d'utiliser l'amphotéricine $\mathrm{B}$ à des doses plus élevées et pour une durée plus prolongée avec une survie meilleure $(67 \%$ versus $39 \%$ avec la forme désoxycholique) (3).

La dose actuellement recommandée d'amphotercine B liposomale est de $5 \mathrm{mg} / \mathrm{kg} / \mathrm{j}$ (1) pour une durée minimale de 12 semaines (4). En effet l'arrêt du traitement antifongique ne peut être envisagé qu'après négativation des prélèvements histologiques (6).

D'autres thérapeutiques sont prometteuses : les imidazolés notamment le posoconazole et le ravuconazole, les échinocandins (caspofungine) et les nouvelles générations de chélateurs de fer, associé à l'amphotéricine B semblent avoir un effet synergique sur la mucormycose nasosinusienne (12). La B-rifampicine semble elle aussi avoir un effet synergique avec l'amphotéricine B (13).

L'apport de l'oxygénothérapie hyperbare et des cytokines (interféron gamma, GMCSF) comme adjuvant au traitement médical est en cours d'étude (1).

La mucormycose nasosinusienne, forme la plus fréquente, est associée à une mortalité élevée (6). Les facteurs de mauvais pronostic sont : le terrain, l'irréversibilité du dysfonctionnement immunitaire (5), le retard diagnostic (au-delà de 6 jours) (5), l'atteinte sinusienne bilatérale, l'extension endocrânienne, orbitaire et au palais $(2,5,6)$ et le traitement exclusivement médical (2). 


\section{CONCLUSION}

La mucormycose nasosinusienne est une infection fongique grave, rapidement évolutive qui survient sur des terrains immuno-déficients et qui peut mettre en jeu le pronostic vital. La guérison et la survie sont nettement meilleures en associant, précocement, un débridement chirurgical du tissu nécrosé à l'amphotericine B liposomale. D'autres thérapeutiques prometteuses semblent potentialiser l'effet de l'amphotéricine B et sont en cours d'évaluation.

\section{REFERENCES}

1- O'Neill B.M., Alessi A.S. et al. Disseminated rhinocerebral mucormycosis: A case report and review of the literature. J Oral Maxillofac Surg ; 2006; 64: 326333.

2- Schütz P., Behbehani J.H. et al. Fatal Rhino-Orbito-Cerebral Zygomycosis caused by Apophysomyces elegans in a healthy patient. J Oral Maxillofac Surg; 2006; 64:1795-1802.

3- Spellberg B Edwards J, Ibrahim A et al. Novel Perspectives on Mucormycosis: Pathophysiology, Presentation, and Management.Clinical microbiology reviews. 2005; 18 3:556- 69.

4- Zeddinia A., Meknia A., Ferchichia L. et al. Mucormycose rhinocérébrale: à propos d'un cas rhinocerebral mucormycosis: a case report . S Médecine et maladies infectieuses ; 2006; $36: 517-19$.

5- Tryfon S., Stanopoulos I., Kakavelas E.et al: Rhinocerebral mucormycosis in a patient with latent diabetes mellitus: a case report. J Oral Maxillofac Surg 2002;60:328-30

6- Vetrone G., Grazi G.L. Ercolani G et al : Successful Treatment of Rhinomaxillary form of mucormycosis infection after liver transplantation: a case report. Transplantation Proceedings. 2006; 38:1445-7.

7-Fairley C, Sullivan TJ, Bartley P. et al. Survival after rhino-orbital-cerebral mucormycosis in an immunocompetent patient. Ophthalmology, 2000; 107:555-
8- Paques M., Wassef M., Faucon B. et al . Mucormycose orbito-sinusienne bilatérale à propos d'un cas. J Fr. Ophtalmol ; 2000; 23 (10) : 1023-1025.

9- Yohai RA, Bullock JD, Aziz AA, et al. Survival factors in rhinoorbital-cerebral mucormycosis. Surv Ophthalmol; 1994; 39: 3-22.

10- Sohail M.A., Al Khabori M. ${ }^{*}$, Hyder J. et al. Acute fulminant fungal sinusitis: clinical presentation, radiological findings and treatment. Acta Tropica. 2001; 80:177-85.

11- Fairley C., Sullivan T.J., Bartley P. et al. Survival after Rhino-Orbital-Cerebral Mucormycosis in an Immunocompetent Patient. Ophthalmology. 2000; 107 (3): 555- 8.

12- Nivoix Y., Zamfir A., Lutun P., Kara F. et al. Combination of caspofungin and an azole or an amphotericin $B$ formulation in invasive fungal infections. Journal of Infection. $2006 ; 52: 67-74$

13- Battikh R., Labbene I., Ben Abdelhafidh N. et al Mucormycose rhinofaciale: à propos de 3 cas. Médecine et maladies infectieuses $2003 ; 33$ : 427-30 